Basrah Journal Of Surgery
Original Article

Bas J Surg, December, 23, 2017

\title{
MICRO-ENDOSCOPIC DISCECTOMY, A MODERN APPROACH FOR LUMBAR DISC PATHOLOGY
}

\author{
Mohamed El Husseini* \& Taghrid Chaaban $^{\circledR}$ \\ ${ }^{*} \mathrm{MD}, \mathrm{PhD}$, Neurosurgeon, Hôpital Libano Français, Zahle, Lebanon. ${ }^{\circledR}$ MS Nursing, IUL, Beirut, \\ Lebanon.
}

\begin{abstract}
In the last decade, the neuro-endoscope has been used increasingly in the surgical management of spinal diseases, both intradural and extradural. Endoscopic discectomy is increasingly performed in lumbar region. This study included 200 patients with lumbar disc pathology operated upon with both techniques: Classic and Endospine Karl Storz system technique introduced by Destandau. Based on results (Macnab modified criteria), microendoscopic discectomy should be used in properly selected patients.

Key words: endoscopic, lumbar disc hernia, Endospine, Destandau technique
\end{abstract}

\section{Introduction}

T umbar disc hernia became a serious

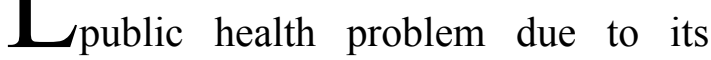
epidemiological, clinical and socioeconomic impact characteristics. In United States it represents the fifth cause of medical service ${ }^{1}$. Because of its increased prevalence among the active population, lumbar disc hernia has an increased economic impact.

Developing of surgical techniques to reduce the costs generated by this disease represents an important aim. In 1991, Foley and Caspart introduced in U.S. the first tubular system for the interlaminar approach of lumbar disc hernia; in 1997, Foley and Smith reported the first microendoscopic discectomy. In 1993, Destandau developed in France the Endospine system and reported the first endoscopic ablation of a lumbar disc hernia $^{2}$. Endoscopic lumbar discectomy has gained increasing popularity among the spine surgeons for the treatment of herniated intervertebral disc largely because it has the ability to overcome the limitation of muscles splitting ${ }^{3}$. The use of an endoscope allows the same access port and the same surgical technique to be used on the vertebral canal and disc while at the same time reducing the skin incision and minimizing the access port. The advantages of this technique are the same as those for discectomy but the immediate postoperative effects are reduced allowing a more rapid rehabilitation and return to previous activities for the patient. Other advantages are a small skin incision with a gentle and minimal tissue dissection and excellent visualization ${ }^{4,5}$.

\section{Patients and Methods}

This study included 200 of patients with lumbar disc hernia operated upon between 2012-2016. One hundred patients had classic open discectomy, and 100 were operated upon by endoscopically endospine method. All patients were re-evaluated at 6 months postoperatively based on Macnab modified criteria and MRI.

Clinically, the patients were re-evaluated at 7 days, 4 weeks and 6 months postoperatively, and for a better appreciation of the results we quantified the persistence of symptoms at 6 months after surgery. We included in this study some factors for better analysis of 
morbidity of lumbar disc hernia, such as: age, gender, home environment, profession, obesity, duration of surgery, quantity of blood lost intraoperatively and postoperatively, complications of each technique, duration of hospitalization, and period of recovery.

The Endospine Karl Storz system consists of an operating tube which is positioned on the vertebral lamina after the incision of the skin, aponeurosis and detaching the muscles from the spinous process. This tube contains four channels for telescope $(4 \mathrm{~mm})$, suction tube (4 $\mathrm{mm})$, surgical instrument $(8 \mathrm{~mm})$ and nerve root retractor. There is an angle of 12 degrees between the working channel and the channel used by the telescope which enables the neurosurgeon to see the tips of the instruments and to use a suction tube as a second instrument. The nerve root retractor allows the nerve to be medialized thus removing it from the operating region. The Hopkins telescope with an angle of 0 degrees, offers a wild field of vision of the operating area. The fiberoptic light cable with a cold light source is integrated in the telescope (fig.1). The entire procedure is performed under constant video-endoscopic control.

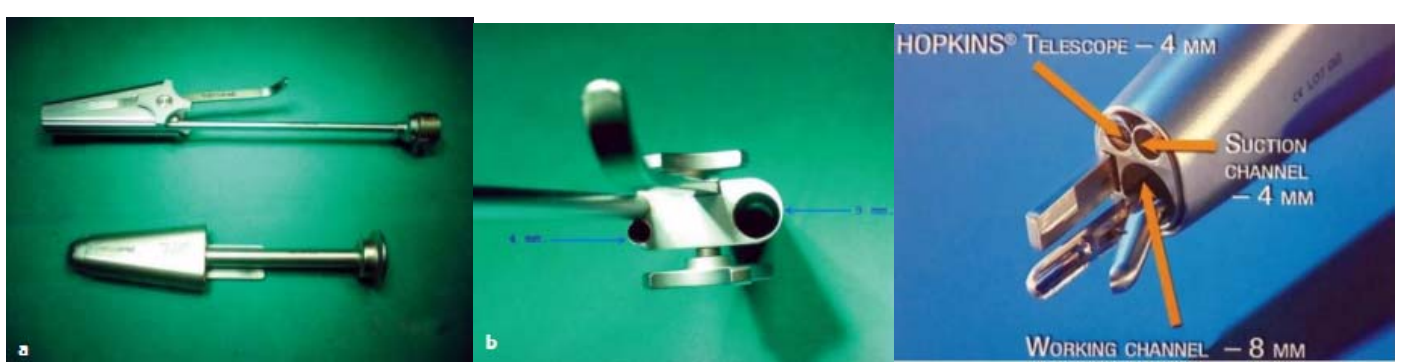

Fig. 1 (a): outer tube and inner tube. (b) inner tube with various parts. Hopkins telescope, working channel and suction channel Endoscopic Spine Surgery-Destandau's Technique' by Rohidass and Destandau.

Under general anesthesia with help of special localization device, and endotracheal intubation, patients were the direction of approach was determined placed in a modified knee-chest position. under fluoroscopic control. The place of The involved disc was localized with the incision was marked on the skin (fig.2).

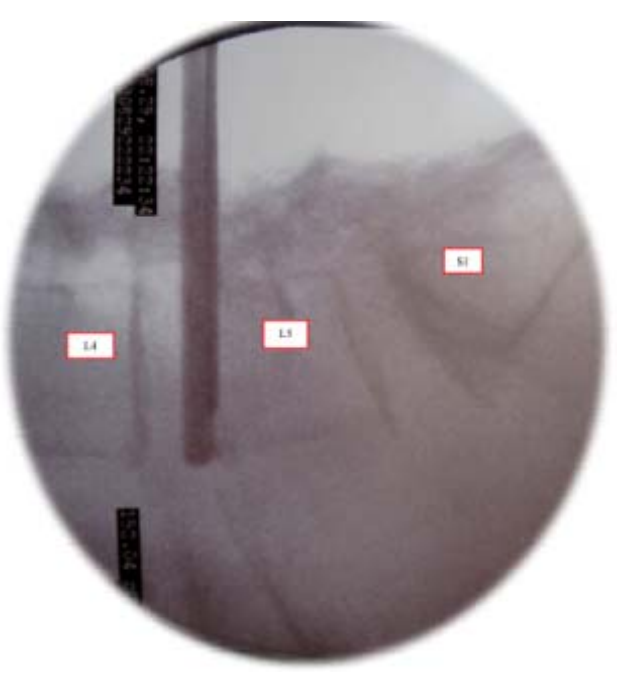

Figure 2: Marking the entry point (fluoroscopic image)

The skin incision was made $5 \mathrm{~mm}$ from the midline at the lateral edge of the spinous process. Hemostasis was carried out by bipolar coagulation. The aponeurosis was incised with scissor at the level of the spinous process from the 
affected levels S1 L5 L4. The paravertebral muscles were spread out and muscles vessels were coagulated with bipolar forceps.

The superior position of Endospine system allowed resection of the lamina and of the yellow ligament and the inferior position allowed zoom effect and facilitated the dissection of the channel (fig. 3, 6).

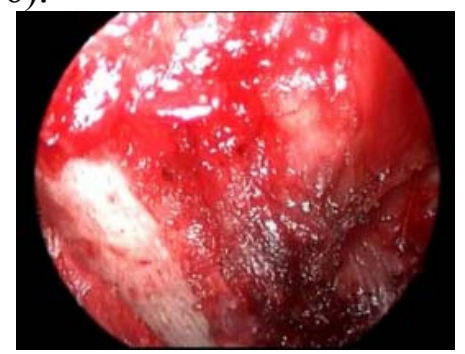

Fig.3: Intraoperative image of the interlaminar space with the superjacent vertebral lamina

Under permanent visual control, a fenestration was made using a Kerrison bone punch ( 2 and $3 \mathrm{~mm}$ ) to detach the yellow ligament and expose dura mater (fig.4\&5). A cotton swab was slid between lamina and dura mater. Also, a part of the articular process was resected to realize an adequate lateral exposure. Bone hemostasis was done with wax.
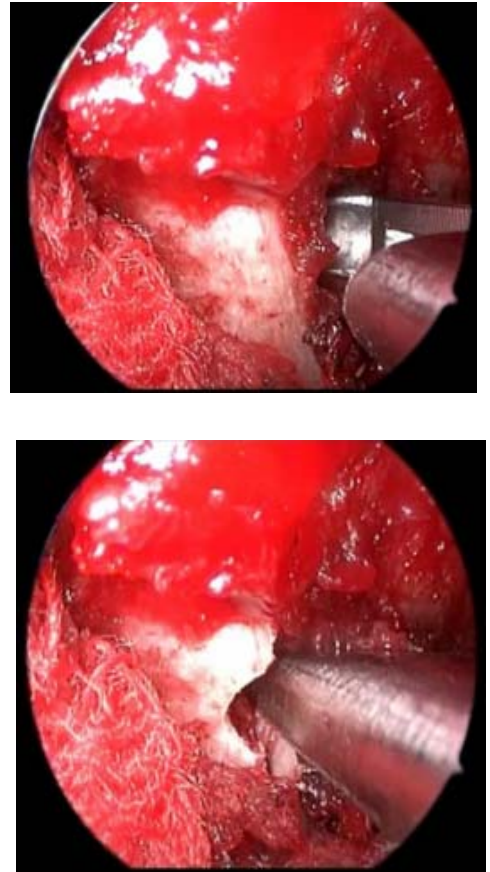

Fig.4\&5: Intraoperative, the bone fenestration
The resection of yellow ligament allowed exposure of the affected nerve root.

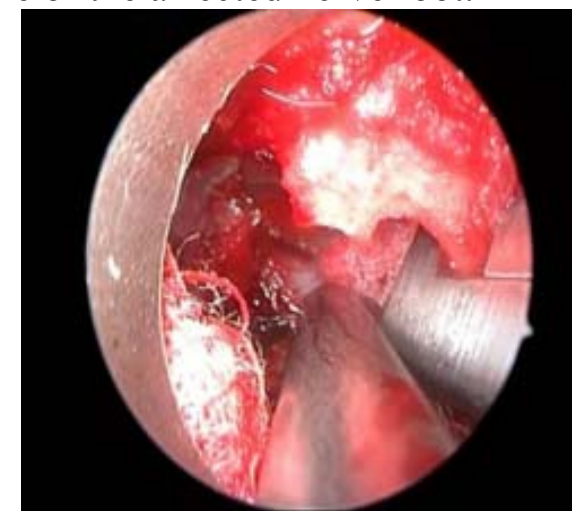

Fig.6: Resection of yellow ligament

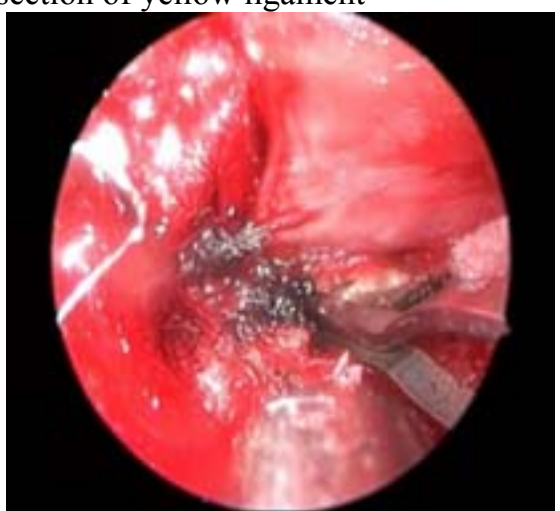

Fig.7: Control of hemostasis periradicular with oblique forceps

The nerve root was retracted medially with a special endoscopic retractor thus exposing the hernia and intervertebral space. A cotton swab was placed to allow medial retraction of the nerve root and removal of the nerve root retractor in order to regain mobility (fig.8).

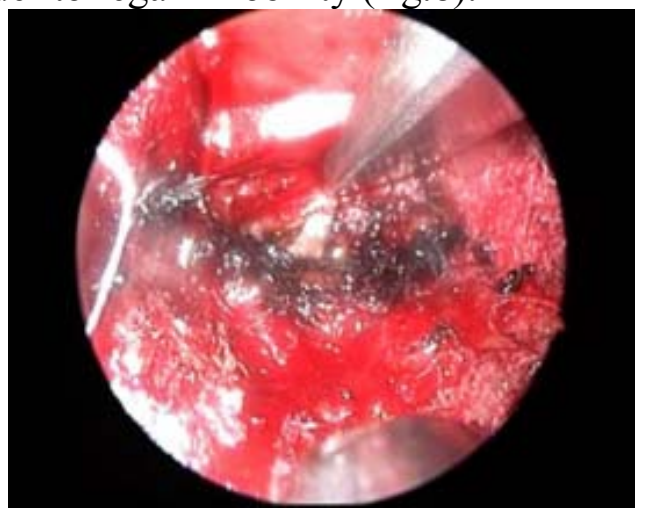

Fig.8: Retraction of the nerve root

An incision of the posterior longitudinal ligament was made and a partial nucleotomy was done (fig.9\&10). 


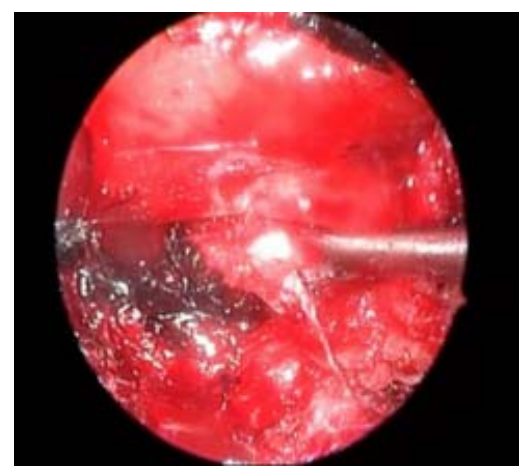

Fig.9: Removal of adherences between disc hernia and nerve root

After exploration of intervertebral space and foramen for any fragments, hemostasis of the muscle vessels was

\section{Results \& Discussion}

This study revealed that maximal incidence was noticed among the active adult population. Eighty percent of endoscopic microdiscectomies and 56\% of open discectomies were made in patients aged 30-59 years. Disc hernia was rare in patients of age group 20-29 (5 cases classic discectomies and 3 cases micro-endoscopic discectomies) and, exceptionally, one case microendoscopic discectomy under 20 years, which is consistent with literature (fig.11).

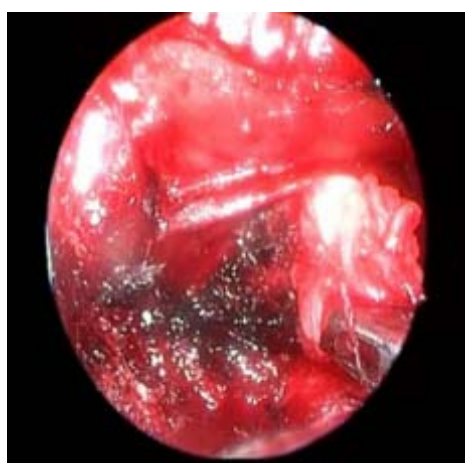

Fig.10: Resection of disc hernia

completed. The Endospine operating tube was removed and the ligament and the skin were sutured.

The increased incidence among 60-69 age group of the patients operated by classic (34\% versus $7 \%$ ) can be explained by associated degenerative pathology (stenosis). The slightly increased incidence of microendoscopic discectomy in patients over 70 years ( $9 \%$ versus $5 \%)$ is also consistent with data. This minimal invasive technique due to reduced resection of the tissues allows a quicker mobilization ( $<24$ hours) and healing of these patients.

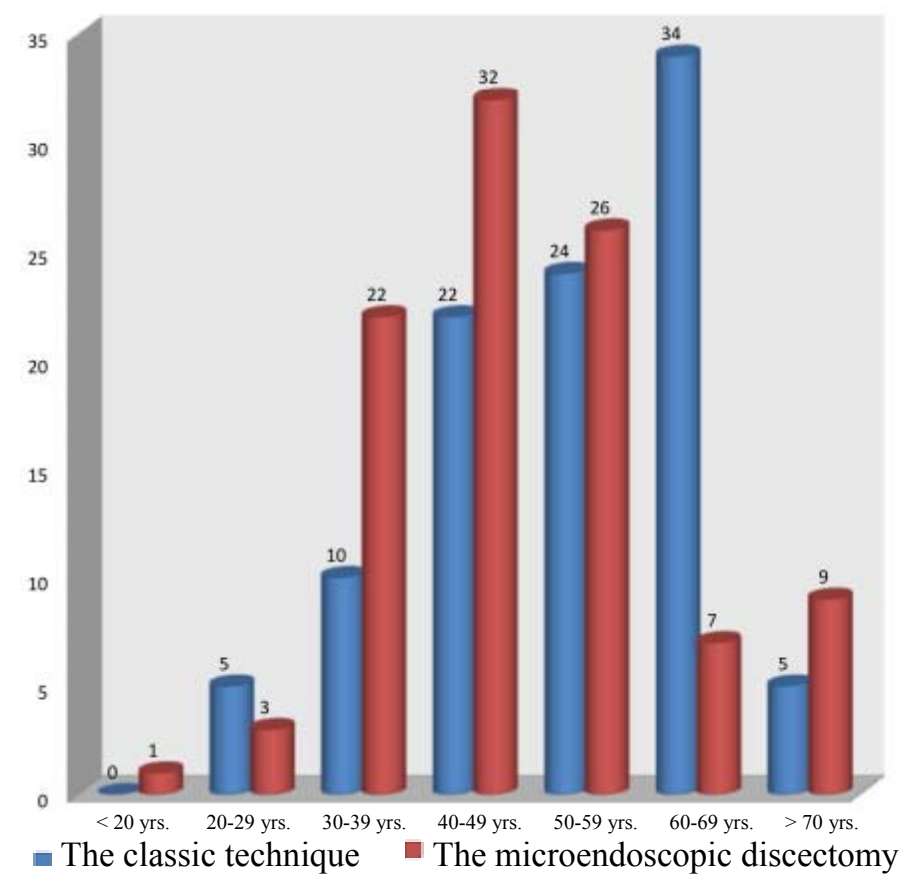

Fig.11: The distribution by age groups 
Regarding gender distribution in this study, the incidence was higher among male patients compared to females in both series (fig.12). In classic technique, the ratio was $2: 1$ for male, while in microendoscopic discectomy, the ratio was $55 \% / 45 \%$, corresponding to those from literature.

There is a male prevalence in lumbar disc hernia operated upon varying from $1.3: 1$ to $2: 1^{6-9}$. Kelsey and Ostfeld bring arguments that there is no "real difference" regarding gender, but male patients are more frequent operated upon due to the type of effort at job and because they cannot wait for favorable evolution with conservatory drug treatment ${ }^{10}$. Other sustain that male patients are more susceptible to disc hernia due to increased disc stress and deficit nutrition of the disc, because male patients have longer paths of diffusionnutrition $^{11,12}$.

The difference may be explained by the height difference which is in absolute value $10.5 \mathrm{~cm}$, female body being $7 \%$ less tall than male body, fact which has consequences at the posterior longitudinal ligament, especially in his lateral part. The height decreases every day by $10-30$ $\mathrm{mm}$ because of pressure given by the weight on the intervertebral discs. The height is greater in the morning, after the night rest compared to the evening, after the daily activity.

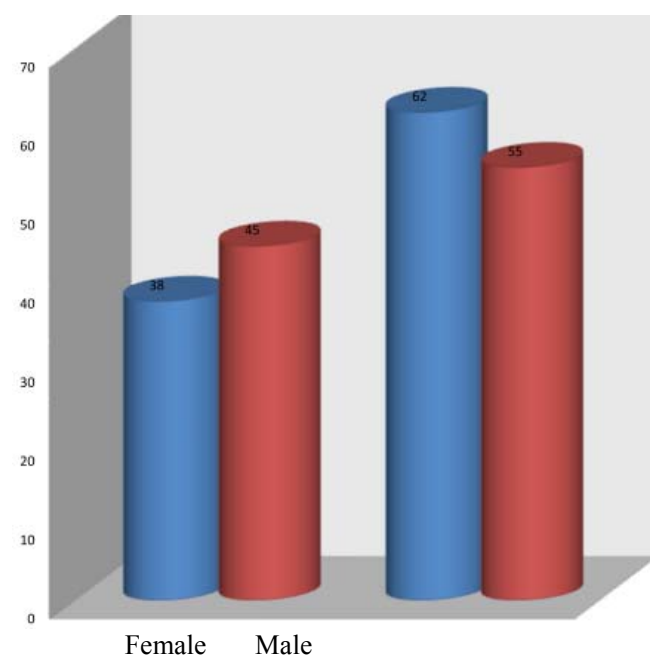

Fig.12: The distribution by gender

Regarding home environment, the prevalence was higher in patients from cities and they had more microendoscopic discectomies (71\% compared to $55 \%$ ).

This study marked an increased percentage of microendoscopic discectomies in educated patients compared with workers due to two aspects: grade of information and education and the intensity of physical work which accelerates the degenerative modifications, plus other pathologies (stenosis, lumbar spondylosis) as demonstrated in table I.

Table 1: The distribution by profession

\begin{tabular}{|l|lc|lc|}
\hline Profession & \multicolumn{2}{|l|}{$\begin{array}{l}\text { The classic technique } \\
\text { (number/percentage) }\end{array}$} & \multicolumn{2}{|l|}{$\begin{array}{l}\text { The microendoscopic discectomy } \\
\text { (number/percentage) }\end{array}$} \\
\hline Workers & 63 & $(63 \%)$ & 44 & $(44 \%)$ \\
\hline Educated & 37 & $(37 \%)$ & 56 & $(56 \%)$ \\
\hline
\end{tabular}

Another aspect we took in consideration was the classification of the patients by BMI (tables II \& III).

Table II: The classification of patients by BMI

\begin{tabular}{|l|l|}
\hline BMI & Patient \\
\hline$<18.5$ & Under weight \\
\hline $18.5-24.9$ & Normal weight \\
\hline $25-29.9$ & Over weight \\
\hline $30-34.9$ & Obese grade I \\
\hline $35-39.9$ & Obese grade II \\
\hline$>40$ & Obese grade III \\
\hline
\end{tabular}


Table III: The incidence of obesity by BMI

\begin{tabular}{|l|l|l|l|l|l|l|}
\hline No. of cases & BMI & BMI & BMI & BMI & BMI & BMI \\
& $<18,5$ & $18,5-24,9$ & $25-29,9$ & $30-34,9$ & $35-39,9$ & $>40$ \\
\hline CD & 0 & 28 & 32 & 29 & 11 & 0 \\
\hline MD & 1 & 30 & 28 & 27 & 13 & 1 \\
\hline
\end{tabular}

CD: classic discectomy. MD: microendoscopic discectomy. BMI: body mass index

Obesity increases the risk of lumbar disc hernia by several mechanisms: excessive biomechanical load, increased total lipids and atherosclerosis which diminishes the transport of nutrients to disc thus favoring degeneration and by secondary inflammation caused by secretion of adipocytes (adipocytokines: adiponectin, leptin and resistin).

In scientific data, the risk of recurrence of operated lumbar disc hernia is 12 times greater in obese patients with $\mathrm{BMI} \geq 30^{13}$.

In this study, like in other similar studies, the location of lumbar disc hernia is represented by L5-S1 and L4-L5: the classic technique $95 \% \rightarrow 46 \%$ L4-L5 and $49 \%$ L5-S1; the microendoscopic discectomy $92 \% \rightarrow 41 \%$ L4-L5 and 51\% L5-S1.
In series with microendoscopic discectomy, the rest of locations were: $8 \%$ (1\% L1-L2, 2\% L2-L3, and 5\% L3L4), while in classic discectomy $5 \%(2 \%$ L2-L3, 3\% L3-L4). The increased percent in microendoscopic discectomy is explained by the fact that patients were from urban medium and the traumatic factor was present in all cases.

Regarding the most affected part, we registered an increased incidence in the left side $(52 \%$ classic, and $62 \%$ microendoscopic), data corresponding with literature and explained by the fact that right handed flip the lumbar column toward right and thus load in lumbar segment, in left.

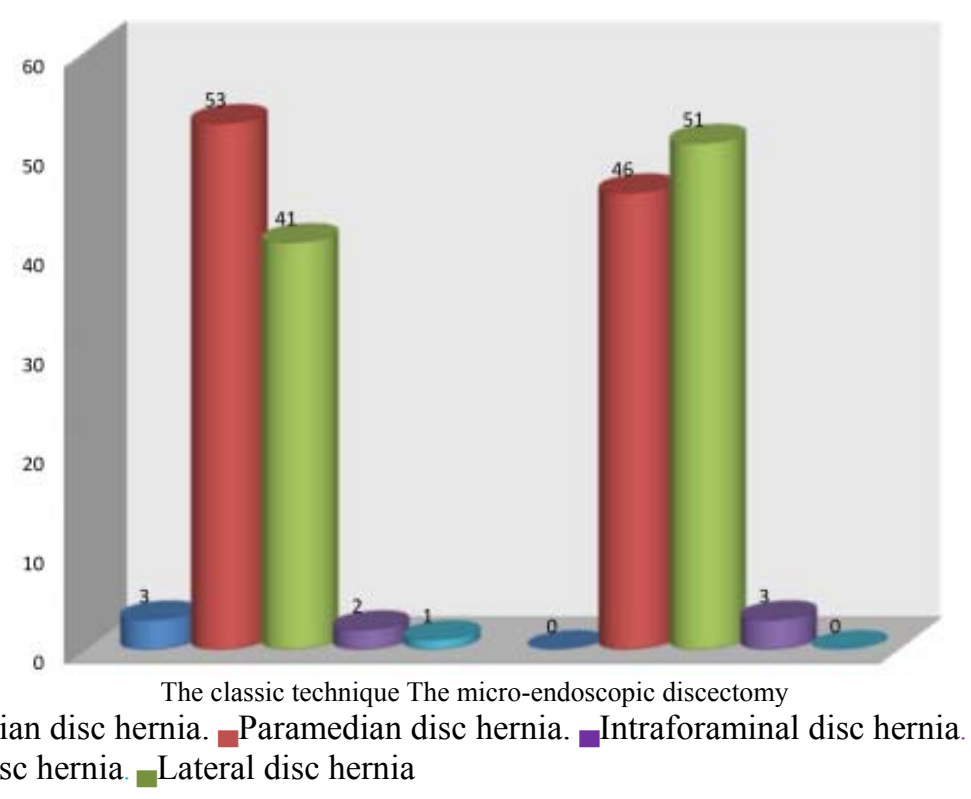

-Dorsal -median disc hernia. $\quad$ Paramedian
Intradural disc hernia. _Lateral disc hernia

Fig.13: Classification of the disc hernia by anatomy-topography

In this study, we had 3 patients with dorsal median disc hernia operated upon with classic, because they required a large decompression of the nerve elements, difficult to realize by endoscopy (fig.13). The paramedian disc hernia requires regarding its size unilateral or bilateral decompression, the classic approach allowing a larger opening. The lateral disc hernia compresses unilateral nerve root and election method was microendoscopic discectomy $(51 \%$ versus 
41\%). The intra-foramina disc hernia compresses the nerve in foramen and microendoscopic discectomy is preferred for a reduced bone resection thus preventing destabilizing spinal cord. The intradural/intraradicular disc hernia has an incidence $0 \%-1 \%$, like data mentioned in literature $0,04 \%-1,1 \%{ }^{14,15}$.

Although the duration of approach is greater for endoscopic technique (90 $\mathrm{min}$ ) compared to classic $(60 \mathrm{~min})$, loss of blood is less in endoscopic $50 \mathrm{~mL}(30-70$ $\mathrm{mL})$ than in classic $(160 \mathrm{~mL})$ and there were fewer complications $(2 \%)$, as it is mentioned in literature.
Regarding the postoperative symptoms, the classic technique was criticized ${ }^{16-21}$. The postoperative evolution based on modified Macnab criteria (excellent evolution: no pain, no restriction of movement; good evolution: intermittent lumbar pain, patient returned to job of low intensity; satisfactory evolution: neurologic deficit but improved, patient need retirement; unfavorable evolution: persistence of neurologic deficit, patient needs new intervention or classic) recommend the Endospine method (fig.14).

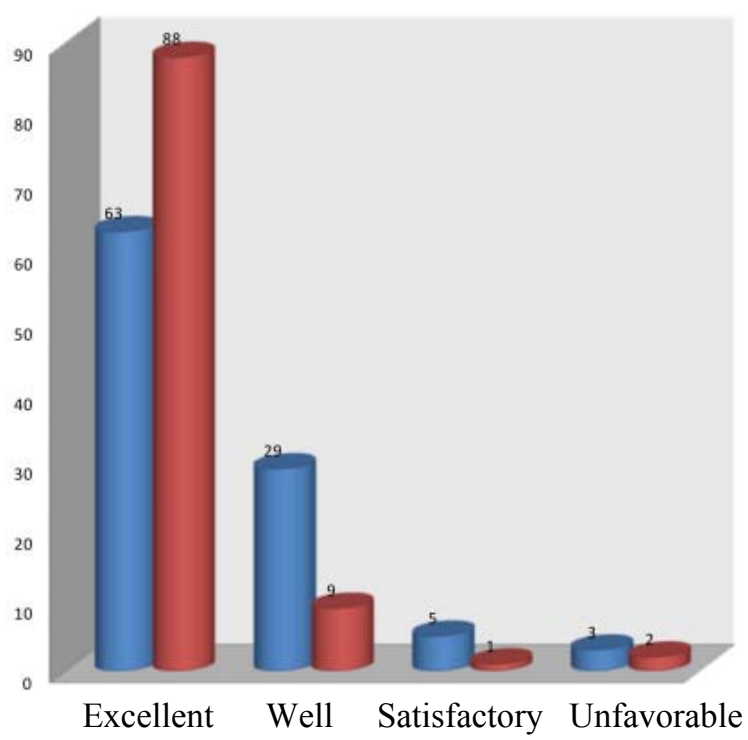

The classic technique. The micro-endoscopic technique

Fig.14: Evolution of the patients (modified Macnab criteria)

In conclusion, the use of the Endospine system in surgical treatment of lumbar disc hernia does not modify the surgical indications. The careful selection of the patients is the key of the excellent postoperative evolution. In this minimally invasive technique it is important to know the location of the affected level in two steps (preoperative and intraoperative) in order to avoid wrong approach. The better visualization offered by the endoscope must be fructified by the surgeon to realize a less aggressive manipulation and resection thus preventing appearance of adherence postoperatively and extended bone resection, a possible source of instability. Another advantage is lower rate of complications (4\%) compared to classic $(10 \%)$. It also reduces the hospitalization and cost, which reduce the socioeconomic impact of this pathology. The initial and long-term results are very good for endoscopic lumbar discectomy, so in selected patients we recommend this method. 


\section{References}

1. Dickman RD, Zigler JE. Discogenic back pain. In: Spivak JM, Connoly PJ, eds. Orthopaedic knowledge update: Spine 3, Rosemont, IL: North American Spine Society, 2006: 319-329

2. Destandau J. Technical features of endoscopic surgery for lumbar disc herniation: 191 patients. Neurochirurgie. 2004;50:6-10

3. Smith MM, Foley KT. Microendoscopic discetomy (MED): surgical technique and initial clinical results; Proceedings of the Thirteen annual Meeting of the joint section of Disorders of the Spine and Peripheral Nerve of the American Association of Neurological Surgeons; 1997 Feb; Newport Beach, CA.

4. Destandau J. A special device for endoscopic surgery of lumbar disc herniation. Neurol Res. 1999;21:39-42

5. Roshan B, Bhavna K, Kisan P.Long-term results of endoscopic lumbar discectomy by "Destandau's Technique" Asian Spine J. 2016 Apr; 10(2): 289-297

6. Bruske-Hohlfeld I, Merritt JL, Onofrio BM, et al. Incidence of lumbar disc surgery: a populationbased study in Olmsted County, Minesota, 1950-1979. Spine 1990; 15:31-35

7. Kelsey JL, Githens PB, O’Conner T, et al. Acute prolapsed lumbar intervertebral disc: an epidemiologic study with special reference to driving automobiles and cigarette smoking. Spine 1984; 9:608-613

8. Spangfort EV. The lumbar disk herniation: a computer -aided analysis of 2504 operations. Acta Orthop Scand Suppl 1972; 142: 1-95

9. Weber H. Lumbar disk herniation: a controlled, prospective study with ten years of observation. Spine $1983 ; 8: 131-140$

10. Kelsey JL, Ostfeld AM. Demographic characteristics of persons with acute herniated lumbar intervertebral disk. J Chronic Dis 1975; 28: 37-50

11. Miller JA, Schmatz C, Schultz AB. Lumbar disk degeneration: correlation with age, sex and spinal level in 600 autopsy specimens. Spine 1988; 13: 173-178

12. Nachemson A, Lewin T, Maroudas A, Freeman MA. In vitro diffusion of dye through the end-plates and the anulus fibrosus of human lumbar inter-vertebral disks. Acta Orthop Scand 1970; 41: 589-607

13. Meredith DS, Huang RC, Nquyen J, Lyman S. Obesity increases the risk of recurrent herniated nucleus pulposus after lumbar microdiscectomy. 2010; 10/7: 575-580

14. Ciapetta P, Delfini R, Cantore GP. Intradural lumbar disc hernia:description of three cases. Neurosurgery, 1981; 8: 104-107

15. Kataoka O, Nishibayashi Y, Sho T. Intradural lumbar disc herniation: Report of three cases with a review of the literature. Spine, 1989; 529-533

16. Caaragee EJ, Han MY, Suen PW, Kim D. Clinical outcomes after lumbar discectomy for sciatica: the effects of fragment type and annular competence. J Bone Joint Surg. 2003; 85: 102-108

17. Donceel P, Du Bois M. Predictors for work incapacity continuing after disc surgery. Scand J Work Environ Helath. 1999; 25: 264- 271

18. Dzioba RB, Doxey NC. A prospective investigation of the orthopedic and psychological predictors of outcome of first lumbar surgery following industrial injury. Spine. 1984; 9: 614-623

19. Herron L, Turner JA, Novell LA, Kreif SL. Patient selection for lumbar discectomy with a revised objective rating system. Clin Orthop Relat Res. 1996; 325: 148-155

20. Hoffman RM, Wheeler KJ, Deyo RA. Surgery for herniated lumbar discs: a literature synthesis. J Gen Intern Med. 1993; 8: 487-496

21. Kjellby-Wendt G, Styf J.Early active training after lumbar discectomy. A prospective, randomized, and controlled study. Spine. 1988; 23: 2345-2351 